\title{
Introduction to Politiphysics
}

\author{
Gary Gindler \\ e-mail address:ggindler@yahoo.com \\ (Submitted September 11, 2019, updated February 18, 2021)
}

\begin{abstract}
A simple classical mechanics model is proposed to describe the political dynamics of human society. The model provides a mathematical structure for the philosophy of the state-man paradigm. The proposed model borrows many classical mechanical terms and generalizes them into the political domain. The wellknown political terms (like freedom and revolution) are given mathematical definitions. Several abstract but solvable problems are presented to demonstrate the general principles of politiphysics, including the Laffer curve and effects of war, immigration, and random forces on society's momentum of inertia.
\end{abstract}

Keywords: Politiphysics, freedom, inequality, equilibrium, Laffer curve, immigration, war, revolution

PACS: 89.20.-a Interdisciplinary applications of physics; 89.65.Gh Economics; Econophysics, financial markets, business, and management; 89.65.-s Social and economic systems

\section{INTRODUCTION}

The American Founding Fathers viewed individual freedom as the cornerstone of the new American state. They also understood freedom within the framework of the relationship between a state and man (by analogy with the fact that religion is the relationship between God and man). This state-man paradigm [1] formed the basis of the newly established country (although the Founding Fathers did not use this term).

The essence of the superiority of individual freedom and individual good over the public good is the mechanism that led to the transformation of the backward British colonies of North America into the mighty United States of America. However, such an idea, albeit remarkably successful, was never actually formalized.

Indeed, human society assumes that some abstract "freedom" exists, but a loosely defined "freedom" is open to broad interpretations. As a result, the word "freedom" is destined to lose its original meaning, like many other terms that fall victim to the everchanging newspeak. This article attempts to define "freedom" in physical terms, to preserve not just the word but the proper meaning of freedom itself.

Obviously, such a program cannot be realized within well-known scientific subjects like physics or mathematics or even wildly popular (and still exponentially growing) Econophysics. Due to the 
lack of this author's phantasms, let us introduce politiphysics.

The reason to present this new term is quite simple. The well-known term of the last few decades, Econophysics, deals mostly with financial markets by using tools and concepts of mathematical physics. For over a century, popular sociophysics studies social phenomena by using methods of theoretical physics. The only area not covered by physicists so far is politics.

Until the beginning of the $20^{\text {th }}$ century, the standalone science of economics practically did not exist. The branch of science associated with modern economists back then was actually called political economy (well, people a century or two ago knew for sure that political power could be gained by utilizing economic power, and vice versa).

Eventually, political and economic branches of the political economy split, creating two scientific subjects - the science of economics and notprecise-at-all, political science. The absence of any meaningful kind of precision and undisputed lack of predictive power in economics was quite a bothering factor. As a result, Econophysics was born at the end of the $20^{\text {th }}$ century (see excellent review in [2].)
Now it is time for physicists to enter the political arena to study the political ramifications of the economic and material life of human society. In fact, neither econophysicists, sociophysicists, economists, nor political scientists can solve political problems - just ask them what the meaning of "freedom" was to the American Founding Fathers.

Last but not least, we have to mention that, in a sense, politiphysics is reflective of the original science of the political economy a la Adam Smith, but now with mathematical precision.

\section{THE CLASSICAL MODEL}

In order to describe a human society as a whole, we must introduce independent variables. According to the man-state paradigm, such variables should belong to the economic domain. Indeed, the man-state paradigm is based on economic power, i.e., wealth allocation between a population and a state.

It is well known that economic power in human societies sooner or later leads to political power. The precise mechanisms of acquiring political power through economic power are not relevant at the moment. What is relevant is that economic 
power could play the role of the foundational independent variable.

If the wealth of a country that belongs to its people (private wealth) is $W_{p}$ and the wealth of a country that belongs to the government is $W_{g}$, the total country's wealth is $W_{t}=W_{p}+W_{g}$. For the one-dimensional model, the variable $x=W_{p}$ will be used as a primary independent variable. We understand wealth as a sum of money (and other monetary instruments) and assets (physical products which, from time to time, get assigned a monetary value) like in []ㅡ.

By definition, $W_{p}$, generally speaking, is bound by $0 \leq x \geq W_{t}$, but cases when $x<0$ and $x>W_{t}$ are possible too.

Condition $x<0$ means private wealth $W_{p}$ is negative, i.e., the country's population as a whole is in debt to the government (financial enslavement or outright slavery or serfdom.)

Condition $x>W_{t}$ means the entire wealth of the country belongs to its citizens, and there is nothing left to the government. In other words, the government wealth $W_{g}$, in this case, is negative, i.e., government (or rather whatever is left of it) functions by borrowing funds from some internal or external sources. If the source of borrowing is internal (their citizens), the total wealth of society does not change; however, it does change if the sources are from other governments or foreign banks, for example.

Speed of wealth reallocation between people and their government $v=d x / d t$ will also play a role in our considerations.

Let us assume the wealth reallocation of a country could be described as a movement of a classical particle in a potential field $U(x)$ that might look like in Fig. 1.

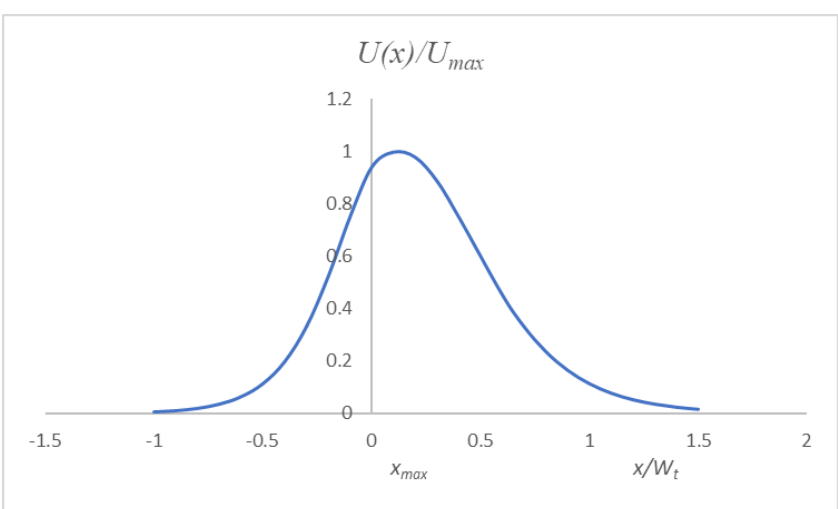

Fig. 1. The normalized bell-shaped potential field of human society.

In the case of $x / W_{t} \leq 0$ the entire wealth of a country belongs to the government (this situation is commonly described as totalitarianism or autocracy.)

Please note we are not considering the case $W_{t}<0$ because it means the entire society exists 
in the form of a puppet state of some foreign power (a typical example would be Indian reservations in the United States.) It does not make sense to consider such societies separately from their masters. In other words, we always assume that the total wealth of society is not negative.

The case $x / W_{t} \geq 1$ means government allocation of wealth is zero, i.e., absence of the traditional government (this area is known as anarchy, but we better call it chaos in order not to incite the modern ideologues of anarchism.)

The potential energy of the society reaches its maximum $U_{\max }$ at some point $x_{\max }$ when $x$ is near zero and reaches its minimum when $x$ approaches $\pm \infty$.

The maximum of a society's potential energy is associated with totalitarian and autocratic states because their oppressive governments can concentrate (by force, in most cases) human activities in a particular area (like wars or scientific research.) The lowest potential energy of a country is either during anarchy $\left(x \approx W_{t}\right)$ or total enslavement or imprisonment of the population $(x<0$.
In the presence of the potential field $U(x)$, classical mechanics [4] defines a function called force $F$ :

$$
F=-\frac{d U(x)}{d x}
$$

In politiphysics, we postulate that this force is a force of freedom or simply freedom. Literally, freedom in politiphysics is the (first) derivative of a country's potential. The typical form for the freedom $F$ is depicted in Fig. 2.

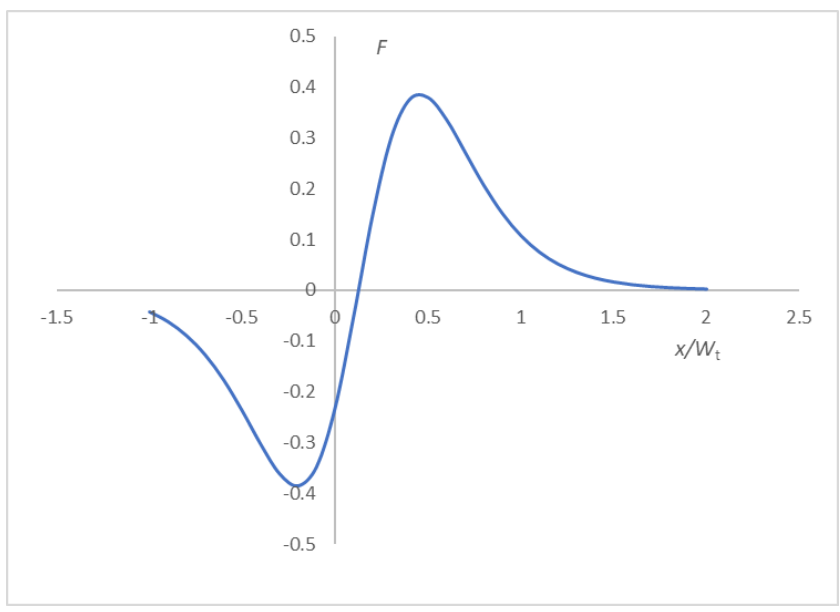

Fig. 2. Force of freedom in a society.

At a certain point, freedom $F$ reaches its maximum $F_{\max }$. As $x$ goes to the left toward 0 (i.e., country moves toward totalitarianism), freedom falls to 0 , and then near $x \approx 0$ becomes negative. We interpret this as enslavement or imprisonment of the entire population by the government. The state of the society under such conditions resembles a concentration camp on a 
nationwide scale. There are many examples of such societies in human history - North Korea or communist Cuba, for example.

If the system moves to the right from the point of maximum freedom $F_{\max }$, freedom also falls (in this case, asymptotically) to zero, even if citizens own the entire wealth of the country.

A well-known example of such societies was realized in some parts of South Russia (modern Ukraine) in the wake of the post-communist revolution's bloody civil war of 1917-1922. Some prominent anarchists established states there, which consisted of numerous farm families loosely interacting with each other and with the outside world. The wealth belonged exclusively to the people all right, but people's lives resembled self-imprisonment behind the walls of their farmhouses. The formal government, law, and order were absent, but the freedom, understood in terms of the state-man paradigm, was absent too.

In other words, a move to the right from the point of maximum freedom $F_{\max }$ usually leads to the degeneration of the government; the move to the left from the point of maximum freedom $F_{\max }$ usually leads to the degeneration of the population.
This conclusion is not new. In fact, it is well known to any person interested in political science where the "left" and the "right" have a well-known and quite important meaning (which is not settled in political science to this day, by the way.) The remarkable thing is that we derived such a conclusion by using a very primitive model from classical mechanics.

Intuitively, it is clear that in the described model, the human society, if left to its own devices, tends to slip into chaos (anarchy) and remain in this state indefinitely. Alternatively, human society can enter the state of totalitarianism, when $x<x_{\max }$, indefinitely.

The difference is: in chaos, the freedom is positive but negligibly small; in totalitarian societies, the freedom is negative and negligibly small (in politiphysics, we call it a controlled entity, or "unfreedom," but historically it was called slavery, serfdom, etc.)

\section{EXACT SOLUTION}

As an example, consider the potential of a society in a form (see Fig 3)

$$
U(x)=\frac{U_{0}}{\cosh ^{2}\left(x / W_{t}\right)}
$$


The model potential (3.1) is slightly different from the typical potential in Fig. 1 - it is deliberately made symmetrical to illustrate the politiphysical solution better.

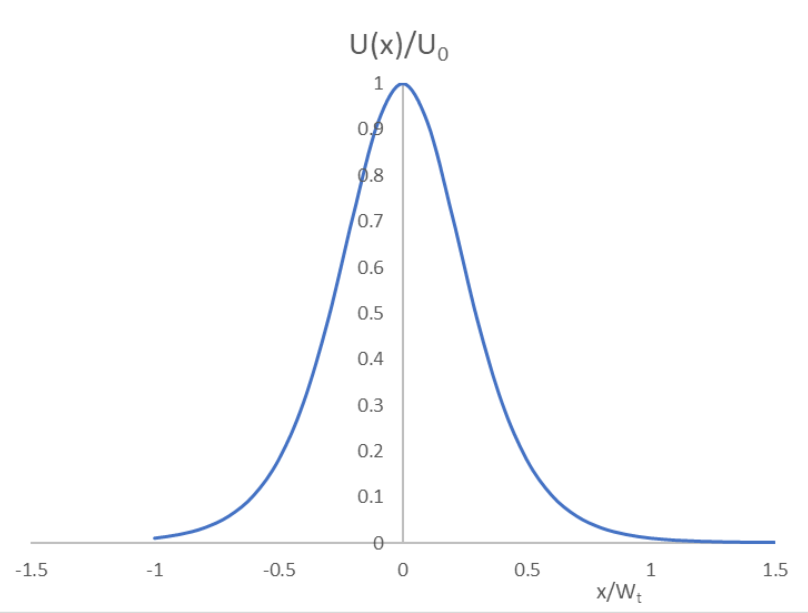

Fig. 3. The normalized exactly solvable potential of society (3.1).

If movement starts at $t=0$ at $x=0$ with zero speed (i.e., with total energy $E=U_{0}$ ), the first integration of Newton's equation

$$
t= \pm \sqrt{\frac{m_{t}}{2}} \int_{0}^{x} \frac{d x}{\sqrt{E-U(x)}}
$$

leads to the exact solution for the potential (3.1):

$$
x= \pm W_{t} \operatorname{arcsinh}\left[\exp \left(W_{t}^{-1} \sqrt{\frac{2 U_{0}}{m_{t}}} t\right)-1\right]
$$

where $m_{t}$ - total "mass" of the society, i.e., total population.

As is would be expected, the movement is symmetrical, and it has a characteristic time

$$
\tau=W_{t} \sqrt{\frac{m_{t}}{2 U_{0}}} .
$$

The force of freedom for potential (3.1) is

$$
F=2 \frac{U_{0}}{W_{t}} \frac{\sinh \left(x / W_{t}\right)}{\cosh ^{3}\left(x / W_{t}\right)} .
$$

Freedom and "unfreedom" (3.4) reach their extremums

$$
F_{\text {max }}= \pm \frac{4}{3^{3 / 2}} \frac{U_{0}}{W_{t}} \approx \pm 0.76 \frac{U_{0}}{W_{t}}
$$

at

$$
x_{\max }= \pm \frac{1}{2} W_{t} \ln (2+\sqrt{3}) \approx \pm 0.65 W_{t}
$$

Because potential (3.1) is symmetrical, forces of unfreedom are negative for $x<0$. The movement in both directions is infinite; the point of maximum freedom or unfreedom is reached at the time

$$
t_{\text {max }}=\ln \left[\sinh \left(\frac{1}{2} \ln (2+\sqrt{3})\right)+1\right] \tau \approx 0.93 \tau
$$

For large $x$, asymptotic of the solution (3.2) is 


$$
x= \pm \frac{W_{t}}{\tau} t
$$

In other words, for the large $t \gg \tau$ physical system moves at a constant speed $v_{\infty}=W_{t} / \tau$.

The reason we introduced such a degenerate example is not an attempt to describe a political system with any degree of precision but rather to enhance the proposed narrative by analyzing the dimensions of physical quantities in the model.

So, $x=W_{p}$ and is measured in units of wealth, like dollars. The variable $m$ is a measure of the "mass" in a country and should be associated with the number of people or simply persons. If we would leave the unit of time the same as in physics, seconds, the speed $v$ is measured in dollars per second, and the units of society's energy $E$ will have dimension

$$
\left[\operatorname{person} \frac{\$^{2}}{\mathrm{sec}^{2}}\right]
$$

Compare the politiphysical energy units with the units of energy in physics:

$$
\left[k g \frac{m^{2}}{\sec ^{2}}\right]
$$

Finally, the force of freedom in politiphysics is measured in:

$$
\left[\operatorname{person} \frac{\$}{\mathrm{sec}^{2}}\right] \text {. }
$$

The model we considered so far was deliberately very simplistic. The model was introduced just to demonstrate the universe of the problems politiphysics is dealing with, and for introducing some new terminology. Nevertheless, the model correctly described the a priori, a well-known concept of freedom, as a driving force of human society in measurable, economic terms.

The more realistic models must acknowledge that any human society is an open (in physical terms), non-conservative (again, in physical terms) system. In other words, any human society is not isolated; there is an external world that interacts with society in various ways.

\section{NON-CONSERVATIVE MODEL}

Under no circumstances, any human society, even very isolated ones (like Aboriginals on tiny Pacific atolls), is left alone to its own devices. Any human society absorbs the energy from external sources (like the Sun, which delivers about 1000 Watts per square meter at Earth's surface) and is influenced by forces of nature 
(some of them are positive, like the Nile's floods, and some are destructive, like hurricanes).

Sometimes this external energy has an immediate effect (weather), and sometimes the effect on human society is delayed by millions of years (oil and coal). Moreover, of course, there are interactions with other human societies, which could impose positive (like trade) or negative (like war) forces.

What state of society is mostly influenced by external forces? The external forces would produce a maximum effect in the areas where the internal force (freedom in politiphysical terms) is weak, i.e., in the areas of $x \approx 0$ and $x \approx W_{t}$. For example, the anarchist states (similar to the described above in Ukraine) are attractive takeover targets for the neighboring states (in fact, those anarchist states in Ukraine did not last for long.)

If anarchists' societies could not do much to resist an external hostile takeover, the totalitarian societies can resist (or make a significant effort to resist) external forces. That is why the society must overcome a certain potential barrier at $x_{\max }$ in Fig. 1 while moving from the left to the right, from totalitarianism/autocracy to a society with some positive freedom.
The original model (3.1) is, of course, quite different from reality. We all know that states with some degree of positive freedom or negative freedom do exist, and they exist for quite prolonged periods of time. In other words, society's one-dimensional movement along the $x$ axis sometimes comes to a complete stop.

Of course, one can modify the potential function $U(x)$ by placing a hard-reflecting barrier at some point $x_{b}$ where $U\left(x_{b}\right)=+\infty$ (or just sufficiently large.) Such a model, under certain conditions, would even permit endless oscillating motion of the society from unfreedom to freedom states.

However, the model that treats human society into a particle in the potential well would be nothing more than the proverbial "curve fitting" (see interesting discussion in [9].) One must admit that in reality, human society is (from the physical point of view) an open system that receives some energy from the outside world and may convert it into some other forms of energy. Also, we must introduce some (non-conservative from the physical standpoint) dissipative forces to be present.

Let us assume the external energy absorbed by human society can be transformed into some rotational energy so that the total kinetic energy $K$ would take the form: 


$$
K=\frac{m_{t} v^{2}}{2}+\frac{I \omega^{2}}{2}
$$

where $I$ - society's moment of inertia, and $\omega-$ the angular velocity.

Under such assumptions, a more complex model would resemble a rotating wheel in a potential field $U(x)$. If we use the mechanical analog again, the wheel on Fig. 1 could, for example, rotate counterclockwise and climb up from right to left (imagine a car running uphill, for example.)

Total energy $E$ of the system is given by

$$
E=U(x)+\frac{m v^{2}}{2}+\frac{I \omega^{2}}{2}+E_{e x t}-E_{\text {diss }},
$$

where $E_{\text {ext }}$ - external energy, pumped into the system, and $E_{\text {diss }}$ - dissipative energy of "friction."

We do not know for sure the exact mechanism of transforming the external energy $E_{\text {ext }}$ into the rotational energy within the society; we just assign this property of the society in such a way it could be described in terms of spinning. Starting with Pauli, such ad hoc considerations are not new in physics.
To visualize the picture further, imagine a car running up a slippery hill. Under certain conditions, like the elevation angle, the mass of a car, and level of friction between wheels and the road, the car would stop while the engine is still running, and wheels continue spinning. The friction forces would fully compensate for the force of gravity.

The stability of this model mandates the external source of energy. In other words, to maintain a certain positive level of freedom, the proper amount of energy must be pumped into the system.

Of course, the model is unstable without a great deal of political will. As President Ronald Reagan said, "Freedom is never more than one generation away from extinction. We did not pass it to our children in the bloodstream. It must be fought for, protected, and handed on for them to do the same."

Obviously, the energy required to sustain a level of positive freedom would be maximal near the point of maximum freedom and minimal in the areas of unfreedom or chaos.

The postulate of the spinning part of the kinetic energy of the society leads us to assume the non- 
uniform internal structure of the rotating body (i.e., society). The moment of inertia of society, like in physics, is a measure of economic diversity - the wealth $W_{p}$ is not concentrated in the dimensionless dot; human society is not an abstract dot without any internal structure.

On the contrary, people's wealth is distributed in such a way that human society more resembles a rotating non-uniform disk than a dimensionless dot. Such economic inequality (several orders of magnitude are not uncommon) allows the external forces to easily transfer the external energy into the rotational kinetic energy of the society. Finally, here is one more argument in favor of the non-conservative (in physical terms) model: we deal with the wealth here, but wealth, as it was shown in [3] , is not conserved, while money certainly is.

\section{ROTATIONAL INERTIA OF SOCIETY}

The physical moment of inertia $I$ is a function of mass distribution in a physical body. In politiphysics, the moment of inertia $I$ is a function of the financial distribution (i.e., wealth distribution) of people within the society.
Let us assume the society (as a disk) has a wealth distribution function (see Fig. 4)

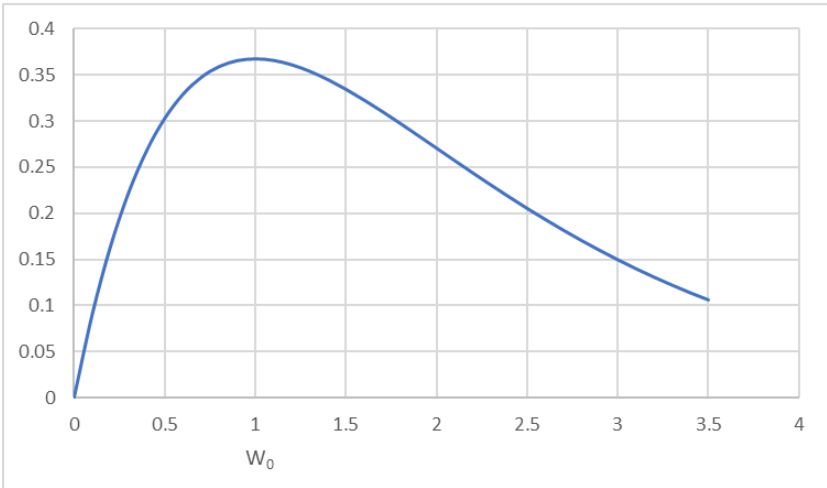

Fig. 4. The wealth distribution function for the model (5.1).

$$
\rho(w)=\frac{m_{t}}{W_{0}^{2}} w \exp \left(-\frac{w}{W_{0}}\right),
$$

where $W_{0}$ - some arbitrary scaling parameter, measured in dollars (it corresponds to the maximum of distribution (5.1), but it is just a coincidence,) and normalization factor $m_{t} / W_{0}^{2}$ is chosen as:

$$
\int_{0}^{\infty} \rho(w) \partial w=m_{t}
$$

The wealth distribution function (5.1), of course, does not have a power-law like the classical Pareto distribution [6]. For example, it does not have sufficiently long power tails, but it serves the purpose of demonstrating the foundations of politiphysics. Besides, there is evidence [11] that income distribution in the United States deviates 
from the Pareto power-law (at least for moderate incomes) and fits exponential distribution (5.1).

Note that the unit of measurement of the wealth distribution $\rho$ is [person $/ \$]$. Total private wealth $W_{p}$ for the distribution function (5.1) is:

$$
\int_{0}^{\infty} w \rho(w) \partial w=2 m_{t} W_{0}
$$

The moment of inertia $I$ for the society $(5.1)$ is [ $[\underline{]}]$

$$
\int_{0}^{\infty} w^{2} \rho(w) \partial w=6 m_{t} W_{0}^{2}
$$

In politiphysics, the moment of inertia $I$ is measured in [people $\left.\cdot \$^{2}\right]$ (compare it with the moment of inertia in physics having units $\left.\left[\mathrm{kg} \cdot \mathrm{m}^{2}\right]\right)$.

Comparing the moment of inertia (5.3) with the moment of inertia of a mechanical disk of mass $m_{t}$ and radius $R, I=m_{t} R^{2} / 2$, we can compute the effective radius $R_{\text {eff }}$ of the politiphysical disk (5.1), $\quad R_{\text {eff }}=2 \sqrt{3} W_{0}$. As in physics, human society's moment of inertia $I$ is linear on a number of people $m_{t}$ and depends quadratically on the characteristic "size" $R_{\text {eff }}$ (in dollar terms) of the society.
Increasing parameter $W_{0}$ makes the distribution function (5.1) wider (while the total wealth also increases.) Decreasing $W_{0}$ produces a concentration of wealth for the poor segment of the society.

The rotational inertia of a disk with long "tails" of mass (i.e., wealth) distribution (5.1) is larger than a uniform disk of the same mass. In other words, societies with a high degree of peoples' inequality are "less dynamic." Such societies are more stable and less sensitive to external forces. It applies both to societies with positive and negative freedom. The only difference is that "spinning" in the case of positive freedom is mostly counterclockwise, while unfreedom is mostly clockwise.

To demonstrate how tails of a wealth distribution affect the moment of inertia of society, consider the following wealth distribution function:

$$
\rho(w)=2 \frac{m_{t}}{W_{0}^{2}} w \exp \left[-\left(\frac{w}{W_{0}}\right)^{2}\right]
$$

The wealth distribution function (5.4) has much shorter tails than distribution (5.1).

Total private wealth of society (5.4) equals (see [5]) $\sqrt{\pi} m_{t} W_{0}$. It is slightly less than the wealth of 
society (5.1). The moment of inertia for distribution (5.4) is $I=m_{t} W_{0}^{2}$. Comparing to the earlier case (5.1), it is six times less. However, this comparison needs to be clarified. Both distributions (5.1) and (5.4) are normalized to the total number of people (5.2), but the scaling parameters $W_{0}$ could be different.

Let us demand not only normalization by the number of people $m_{t}$ but also normalization by the total wealth. If $W_{0}^{\prime}$ is the effective scaling parameter of distribution (5.4) that produces the same total wealth as distribution (5.1), $W_{0}^{\prime}=(2 / \sqrt{\pi}) W_{0}$, so that $W_{0}^{\prime}=\left(2 / \sqrt{\pi)} W_{0}\right.$.

Comparable to the case (5.1) terms, a moment of inertia of society (5.4) is

$$
I=(4 / \pi) m_{t} W_{0}^{2}
$$

In other words, society (5.4), by all counts - with the same number of people and the same total private wealth - has less rotational inertia than society (5.1) with longer wealth distribution tails, thus wider inequality. Comparing society (5.4) with the uniform wealth distribution, we can obtain its effective "radius" $R_{\text {eff }}=\sqrt{2} W_{0}$.

On a side note, the above introduction of the effective radiuses of societies could lead to a new method of computing wealth inequality. If we introduce "average" wealth as $W_{a}=W_{p} / m_{t}$ and express the effective radius above in terms of $W_{a}$,

$$
R_{e f f}=\gamma W_{a} .
$$

For society (5.1) with longer tails $\gamma=\sqrt{3}$ (i.e., $\gamma>1$ ), and $\gamma=\sqrt{2 / \pi}$ for the society (5.4) with shorter tails (i.e., $\gamma<1$ ). Note that for the uniform wealth distribution, $\gamma=1$.

The friction force in the considered model is proportionate to the $m_{t}$ and the government wealth $W_{g}$. This friction with the government creates the torque that equalizes the force of freedom and stabilizes the system.

Who is dealing mostly with the government? It is the part of the society on the outer banks of wealth distribution, $w / W_{0} \gg 1$, wealthy people. Most of the poor people $\left(w / W_{0} \ll 1\right)$ are acting as "clients" or "subjects" of the government.

The poor segment of society is mostly indifferent to the political processes in that society because their linear velocity is close to zero. Some politicians, however, are capable of shifting the rotational axis of society. For example, they can shift the rotation axis toward the center of mass of the society, known elsewhere as the middle 
class. In such a case, it is the middle class who becomes indifferent to the political processes in a country. They disengage from reality, and only very wealthy people and very poor people interact with the government.

It is well-known that politicians are very often pandering to the middle class. Political pundits are well aware that it has nothing to do with the middle class per se - the goal is not to "help" the middle class in any way, but rather to shift the society's rotational axis from zero to some value close to the center of wealth $W_{c}$.

The moment of inertia of a society spinning around the center of wealth is minimal, and such a society can be more easily influenced, controlled, and manipulated. The reason is simple - if the axis is located at the center of wealth $W_{c}>0$, the poor segment of society gets exposed. Their linear speed is no longer a near-zero; they become active players in the political arena.

The polarization of society is the end result of such a shift. When the rotational axis was at zero wealth, the entire society's spin occurs in the same direction (the angular velocity is the same for everybody, but the linear speed, even if it depends on the rotational radius, i.e., wealth $w$, still has the same direction for everybody.)
If the rotational axis shifts toward the center of wealth $W_{c}$, the linear velocity vector of very poor people starts moving in the opposite direction from the velocity of the very wealthy (compare this situation with the opposite points of a rotating wheel.)

For example, the center of wealth $W_{c}$ for society (5.1) is

$$
W_{c}=2 W_{0}
$$

The moment of inertia $I_{c}$ of society (5.1) with the axis at the center of wealth is:

$$
I_{c}=2 m_{t} W_{0}^{2},
$$

It is three times less than the original (compare to formula (5.3)). (Side note: Huygens-Steiner theorem also true in politiphysics.) If a politician manages to shift the axis of society (5.1) from $w=0$ into the center of wealth $w=W_{c}$, controlling and managing the population suddenly becomes three times easier. As an add-on benefit, the politician can now deploy an army of inexperienced (in politics), poor people by emphasizing their antagonism to the wealthy. Before that shift, the poor were moving in the same direction as the wealthy ones and never felt any antagonism toward them. 
In other words, the $19^{\text {th }}$-century theory of the class-based antagonism now, in politiphysical terms, simply means shifting the rotational axis of a society, which leads to the (desired by some politicians) polarization of society. In other words, politicians are very capable of utilizing wealth inequality of the unsuspecting population as leverage to gain extra political points.

To further illustrate the effects of inequality on society's kinetic energy, consider the degenerate case of the society whose internal structure looks like a barbell:

$$
\rho(w)=m_{1} \delta\left(w-W_{1}\right)+m_{2} \delta\left(w-W_{2}\right),
$$

where $\delta(x)$ - Dirac's delta function, and, for the sake of concreteness, $W_{1}<W_{2}$. That is the ultimate (albeit very abstract) structure of the society where only two groups of citizens exist - the number $m_{1}$ of poor people with average wealth $W_{1}$, and $m_{2}$ the quantity of reach people with average wealth $W_{2}$. The center of the wealth of society $(5.5)$ is at

$$
W_{c}=\frac{m_{1} W_{1}+m_{2} W_{2}}{m_{1}+m_{2}}
$$

The moment of inertia for (5.5) for the arbitrary axis $L$ is

$$
I=m_{1}\left(W_{1}-L\right)^{2}+m_{2}\left(W_{2}-L\right)^{2}
$$

It is easy to show that moment of inertia $I$ (5.6) reaches its minimum $I_{c}$ in the case of spinning around the center of wealth $L=W_{c}$,

$$
I_{c}=\frac{m_{1} m_{2}}{m_{1}+m_{2}}\left(W_{2}-W_{1}\right)^{2}
$$

Formula (5.6) demonstrates another real (albeit always hidden) goal of all politicians - shift the rotational axis either to the poor $L=W_{1}$ or to the rich $L=W_{2}$. By doing so, politicians can nullify the contribution of any desired income group to the rotational kinetic energy of society. The common knowledge is that the politicians, in most cases, do not even realize the underlying reason for their actions - they just act on instinct.

Typical example - Germany's National Socialists in the 1930s. They managed to shift the rotational axis well beyond the center of wealth, thus making the rich people very complacent, converting the poor into a formidable political force, and transforming the middle class into very politically active and loyal supporters. The society got a tremendous rotational catalyst from the confiscated Jewish wealth []ㅡ, and that pushed them very quickly over the top into unfreedom territory. Moreover, due to the high 
angular speed, the rolling over the potential barrier - over $U_{\max }$ on Fig. 1 - was practically effortless. Indeed, the self-enslavement was enthusiastically supported by most of the German population at the time.

Another trick politicians use is advocating for the minimization of inequality between the wealth groups.

Formula (5.7) shows why - the moment of inertia shrinks when financial equality is the declared official goal; it goes to 0 in the case of full equality $\left(W_{1}=W_{2}\right)$. Such (utopian) society can be manipulated, pushed, led, indoctrinated, and controlled ("rotated" in mechanical terms) with ease. Unfortunately, by doing so, politicians remove the only known mechanism of stabilization of society. The fallacy of wealth equality is well-known and very old - it was Plutarch who wrote over two millennia ago that "An imbalance between rich and poor is the oldest and most fatal ailment of a republic."

When society suddenly starts rotating too fast, it could quickly slip into either anarchy or slavery. It did happen in recent human history, when, after dissolving the USSR, Russia got an adrenaline shock of freedom in the 1990s. This shock led Russia away from totalitarianism thru the point of maximum freedom under President Yeltsin.
Russia passed the point of maximum freedom rather quickly and reached the near-chaotic point during the financial crisis of 1998. Then Russia turned back and approached authoritarianism under president Putin, using record-high oil prices as a catalyst for climbing up Russia's potential barrier from right to left in Fig. 1.

Needless to say, part of the stabilization of any society is to take away the political "heat" generated by too much rotational kinetic energy. Such heat sinks are usually played by various humanitarian efforts - libraries, art, museums, fundamental and large-scale scientific projects, etc.

The "heat" produced by "friction" of the society's spin needs thermodynamic considerations, similar to the excellent review in [9].

Finally, we can conclude that both left and right ideologies have a right to exist. From the politiphysical point of view, the left-wing politicians are advocating for low private wealth with respect to total wealth, $W_{p} / W_{t} \ll 1$. The right-wing politicians are advocating for high private wealth with respect to the total wealth, i.e., $W_{p} / W_{t} \sim 1$.

There is another politiphysical difference between the two camps. Politicians from the left 
are trying to shift society's rotational axis from left to right (from the poor to the rich,) and politicians from the right are trying to shift the axis from right to left (from the rich to the poor.)

During this process, the left camp is trying to take the political power from the rich, and the right camp is trying to take political power from the government. In other words, both camps are not fighting with each other per se; they are fighting different battles, but sometimes these battles overlap.

Both ideological camps are doing so to gain political control over the society by nullifying rotational kinetic energy from the target wealth segments. However, due to the wealth inequality, the right ideologies appear to produce more stable societies than the left ones (i.e., societies with larger momentum of inertia.) Indeed, the cemetery of world political history is filled with numerous short-lived leftist societies.

The introduction of the rotational kinetic energy of a society leads to a formal definition of the term "revolution."

Intuitively, everybody understands that any political revolution means a substantial change in private wealth. If the characteristic time for the given society potential $U(x)$ is $\tau$, any revolution mandates

$$
\frac{\tau}{W_{p}} \frac{\partial W_{p}}{\partial t} \gg 1 .
$$

However, the condition (5.8) is just a necessary condition. We have to complement it with another condition

$$
\frac{\tau_{r}}{\omega} \frac{\partial \omega}{\partial t} \gg 1
$$

where $\tau_{r}$ - characteristic time for the rotational component of a society. Finally, the true (in politiphysical terms) revolution needs one more condition - for the shifting of the rotational axis:

$$
\frac{\tau_{r}}{L} \frac{\partial L}{\partial t} \gg 1
$$

The conditions (5.8) - (5.10) represent necessary and sufficient conditions for the revolution in politiphysical terms.

From this perspective, the French Revolution at the end of the $18^{\text {th }}$ century falls in the "true revolution" category, but on the surface, the American Revolution, which happened at approximately the same time, does not. 
In fact, the revolting colonies did satisfy the change in rotation condition (5.9). However, the Founding Fathers left the rotational axis at the same position as the British - near zero, and the wealth redistribution in the original 13 colonies, after the Declaration of Independence was signed in 1776, was rather small. However, at that time, these colonies were part of a different country the British Empire, and for the Empire, all conditions for the revolution had been met.

\section{IMMIGRATION}

The effects of immigration could be demonstrated on the exaggerated dipole model (5.5). If, for example, the total number of poor people suddenly increases,

$$
m_{1} \rightarrow m_{1}+\Delta m_{1}
$$

where $\Delta m_{1} \ll m_{1}$, and the wealth of the group increases in the same proportion,

$$
W_{1} \rightarrow W_{1}+\Delta W_{1}=W_{1}+\Delta m_{1} \frac{W_{1}}{m_{1}}
$$

but the wealth and number of wealthy people do not change, the change to the moment of inertia $\Delta I$ for the dipole (5.5) will be (retaining only terms linear on $\left.\Delta m_{1}\right)$ :

$$
\Delta I \approx \Delta m_{1}\left(W_{1}-L\right)\left(3 W_{1}-L\right)
$$

Expression (6.3) has one trivial and one nontrivial result. The trivial result is that shifting the rotational axis to the poor, $L=W_{1}$, nullifies the rotational effect of immigration completely, as expected. However, the non-trivial result is that such nullification also occurs at the point of $L=3 W_{1}$, i.e., at the position of the non-existent in the model (5.5) middle class with average wealth three times that of the poor.

That qualitatively explains once more the pandering of many politicians to the middle class. Politicians want immigration to expand the taxpayer and voter base, while concurrently trying to make effects of immigration "not visible" to the rest of the population.

Now consider simultaneous changes to the poor and wealthy segments of the dipole society (5.5). Similar to (6.1) and (6.2),

$$
m_{2} \rightarrow m_{2}+\Delta m_{2}
$$

and

$$
W_{2} \rightarrow W_{2}+\Delta W_{2}=W_{2}+\Delta m_{2} \frac{W_{2}}{m_{2}} .
$$

Simple calculation leads to the suggestion that an increase in the immigration of the poor could be 
fully compensated by changing the number of wealthy people by the amount

$$
\Delta m_{2}=-\Delta m_{1} \frac{\left(W_{1}-L\right)}{\left(W_{2}-L\right)} \frac{\left(3 W_{1}-L\right)}{\left(3 W_{2}-L\right)}
$$

Here we need to consider three cases.

(a) $0 \leq L<W_{1}$

In this case (assuming $\left.W_{2}>L\right) \quad \Delta m_{2}$ becomes negative, i.e., the moment of inertia of the society stays the same only if the number of the wealthy people and their wealth decreases. For $L=0$,

$$
\Delta m_{2}=-3 \Delta m_{1}\left(\frac{W_{1}}{W_{2}}\right)^{2}
$$

Note that the square term in brackets above is very small. In this case, wealthy people can offer some resistance to immigration, because such immigration decreases their number and their wealth, albeit in practically negligible terms.

(b) $W_{1}<L \leq 3 W_{1}$

In this case, $\Delta m_{2}$ becomes positive - the number of wealthy people must multiply along with their wealth to negate the effect of immigration. The side effect of this could be, as mentioned before, a politically indifferent middle class.
This situation did happen in the United States when effects of massive immigration from the poor Latin America countries were fully offset by a relatively small number of well-educated Jews fleeing from the crumbling Soviet Union and her satellites in Eastern Europe. A similar process was observed in Israel.

(c) $3 W_{1}<L<W_{2}$

In this case, a change in the number of wealthy people $\Delta m_{2}$ becomes negative again. However, this time, wealthy people, without any hesitation, will revolt against immigration that simultaneously shifts the rotational axis beyond the middle-class area toward them. There are numerous examples of the rich's behavior in this case: they will make substantial efforts to keep the rotational axis of the society in the area around zero (or at least below the poor level $W_{1}$.)

\section{WAR}

It is well known that during a war, mostly poor people die. In politiphysical terms, it means the number of poor people decreases, $\Delta m_{1}<0$, and analysis is similar to the immigration analysis above, but with an inverted sign. For the dipole model (5.5), consider three cases. 
(a) $0 \leq L<W_{1}$

In this case, the change in the number of rich people $\Delta m_{2}$ becomes positive. In other words, war is beneficial to rich people, even if a number of poor people die. However, the entire effect (6.5) is exceedingly small. Analogous to this kind of war would be American involvement in both World Wars.

(b) $W_{1}<L \leq 3 W_{1}$

That is, probably, the evilest scenario for a country. Both poor and wealthy people die during the war while the rotational axis moves toward the middle class, making it politically irrelevant.

This situation did happen in Great Britain during both WWI and WWII. As a result, this doublewar punch reduced not only the number of poor people but disproportionally decreased a high number of the rich British nobleman, too (by the centuries-old British tradition, the rich noblemen's kids were serving mostly in the armed forces.) Because the rotational axis shifted toward the middle class, that made it politically insignificant, and Prime Minister Winston Churchill lost the electoral base and the very first post-war elections of 1945.

(c) $3 W_{1}<L<W_{2}$
That is a very troubling case for the country. The number of wealthy people $\Delta m_{2}$ during the war increases, along with their wealth, while poor people die.

The rich become richer while they enthusiastically support the war, but, at the same time, the rotational axis shifts toward the rich, thus making them politically irrelevant in the end. After this type of war, the entire cohort of wealthy people become political hostage to the all-powerful poor masses. Enter the communist coup in Russia in 1917, which happened even as WWI was still ongoing.

\section{EQUILIBRIUM STATE}

A necessary (but not sufficient, of course) condition for the dynamic equilibrium demands that the force of freedom (2.1) would be equal to the "friction" force.

The friction force, being a force of interaction between the people and the government, should be proportional to the government wealth $W_{g}$, total mass $m_{t}$, and angular velocity $\omega$ :

$$
F\left(W_{p}\right)=-\frac{\partial U\left(W_{p}\right)}{\partial W_{p}}=\mu m_{t} W_{g} \omega^{2}
$$


Where $\mu$-dimensionless friction coefficient. The reason we postulate the quadratic dependence of the angular speed is based on the well-known fact that resistance in air and fluids, at first approximation, is governed by similar law (and, of course, we applied eternally popular in physics the unit of measurement argument.)

From (8.1) follows

$$
W_{g}\left(W_{p}\right)=\frac{F\left(W_{p}\right)}{\mu m_{t} \omega^{2}}
$$

Formula (8.2) gives the amount of government wealth as a function of private wealth. Because the force of freedom near the point of maximum freedom has a shape of the inverted parabola for positive $W_{p}$, government wealth as a function of private wealth has the same shape as in Fig. 2 (we assume that friction, angular velocity, and total population do not change in the vicinity of the equilibrium.)

It is not difficult to see that (8.2) is a close mathematical equivalent of the well-known Laffer curve [7]. (Arthur Laffer is a prominent supply-side economist who gained notoriety as a member of President Reagan's Economic Policy Advisory Board.) However, the original Laffer curve propagates into areas of $W_{p}=0$ and
$W_{p}=W_{t}$, but the formula (8.2) is valid only around some equilibrium state, distant from these points. Besides, the friction coefficient $\mu$, most likely, depends on $W_{p}$ too, and in a non-linear fashion. In fact, it would be very strange if it would not be so.

Moreover, of course, angular speed $\omega$, strictly speaking, should not be considered as an independent variable, especially due to the possible "relativistic" effects for high rotational velocity.

Indeed, society is better modeled not as a rigid body but as an elastic one, where perturbations of the wealth density $\rho(w)$ are disseminated with characteristic speed $c$. For any given angular velocity, $\omega$ the linear velocity for the rich is proportional to the "radius" $w$ and can approach the characteristic speed $c$.

The existence of "relativistic" effects in human society is a fascinating perspective because such effects should be observable (for example, "Mach waves.") Also, in elastic bodies, different points could rotate at different angular velocities, i.e., rotation of points far away from the axis will be delayed due to the finite propagation speed of wealth perturbations. From such perspective, the observed in [12] variations of the Pareto index 
over time are just manifestations of wealth perturbations ("wealth waves") propagating thru non-uniform media.

We deliberately omit discussions about how society can reach the point of the equilibrium. However, we have to emphasize that if the equilibrium is reached, it requires a continuous supply of external energy to sustain a certain level of positive freedom.

Finally, from (8.2), we conclude that government wealth $W_{g}$ (i.e., taxes government collects) reaches its maximum near the point of the maximum positive freedom of the society. In retrospect, everyone understands that.

\section{RANDOM FORCES}

Let us discuss some of the effects of random external forces on society. We will use the primitive dipole model (5.5) and limit our analysis by studying the effects of the random forces on the rotational inertia of society only.

Assume that the number of poor people and the number of rich people is constant, and their corresponding wealth could be described as

$$
W_{1} \rightarrow W_{1}+\sigma_{1} \delta W_{1}
$$

and

$$
W_{2} \rightarrow W_{2}+\sigma_{2} \delta W_{2}
$$

where $\delta W_{1}$ and $\delta W_{2}$ - random variables with zero mean and unitary dispersion, and amplitudes $\sigma_{1}$ and $\sigma_{2}$ are not negative. Correlation function $R$ between random variables $\delta W_{1}$ and $\delta W_{2}$ is

$$
R=<\delta W_{1} \delta W_{2}>
$$

The brackets in formula (8.1) denote averaging by the ensemble.

In this case, the average momentum of inertia at the center of wealth for the dipole model (5.5) is

$$
\left.<I_{c}>=\frac{m_{1} m_{2}}{m_{1}+m_{2}}\left[\left(W_{2}-W_{1}\right)^{2}+\sigma_{1}^{2}-2 \sigma_{1} \sigma_{2} R+\sigma_{2}^{2}\right)\right]
$$

In the simplest case, when changes in the wealth of the poor and wealth of the rich are not correlated, $R=0$, and

$$
<I_{c}>=\frac{m_{1} m_{2}}{m_{1}+m_{2}}\left[\left(W_{2}-W_{1}\right)^{2}+\sigma_{1}^{2}+\sigma_{2}^{2}\right]
$$

Formula (8.2) differs from formula (5.7) by nonnegative term $\sigma_{1}^{2}+\sigma_{2}^{2}$. In other words, uncorrelated random external conditions increase the center of wealth, i.e., pushing it away from 
the poor and closer to the rich. From the earlier chapters, we know that such an outcome is preferred to the left ideologues, and, lo and behold, we all know that creating unrest is what they often do, but, of course, without a clear understanding of why it helps their cause.

A very interesting case is when the changes in the wealth of the poor and the rich are anticorrelated, $R=-1$. It means that the rich, for example, increase their wealth exclusively at the expense of the poor, and vice versa. In this case

$$
<I_{c}>=\frac{m_{1} m_{2}}{m_{1}+m_{2}}\left[\left(W_{2}-W_{1}\right)^{2}+\left(\sigma_{2}+\sigma_{1}\right)^{2}\right]
$$

This situation is very rare because it assumes total control over the wealth redistribution process. Of course, such indignation and stealing from the poor significantly pushes the rich toward political irrelevance. And vice versa - outcry confiscations from the rich push the poor closer to the irrelevancy.

If the random changes of wealth between the two groups are fully correlated, $R=1$, and

$$
<I_{c}>=\frac{m_{1} m_{2}}{m_{1}+m_{2}}\left[\left(W_{2}-W_{1}\right)^{2}+\left(\sigma_{2}-\sigma_{1}\right)^{2}\right]
$$

If $\sigma_{1}=\sigma_{2}$, formula (8.4) represents a very important President Kennedy case, when "a rising tide lifts all boats." In this case, the second term in (8.4) vanishes, and formula (8.4) transforms back into (5.7).

In other words, in a society where fluctuations of wealth between poor and rich segments are fully synchronized, its rotational inertia does not change, i.e., such a society is theoretically immune from random external influences.

\section{CONCLUSIONS}

The proposed model is so simple that it is perhaps difficult to believe it can contribute that much to our understanding of political behavior. However, there is no compelling reason for repeating the main points of the article. Instead, let us discuss some important topics not included here and future directions.

The classical physical particles, as it is known, are "brainless" in the sense they do not possess "knowledge" about the entire potential field $U(x)$. On the contrary, quantum particles somehow "know" the entire potential field, and that "knowledge" allows them to tunnel through classically prohibited potential barriers. 
Human society (not the classical model above, of course) also, like quantum particles, knows about the entire potential field ahead of them. Without doubt, people (well, some of the people for sure) in the societies found at $x<x_{\max }$ (Fig. 1), i.e., to the left of the potential barrier between unfreedom and freedom, are well aware of the forces of positive freedom that lie to the other (right) side of the potential barrier.

Finally, human society carries so-called long correlations, i.e., humans do remember things, and in many (but not all) situations, political decisions are made based on the lessons learned from the past. In other words, human societies sometimes are acting not only on the demands of the local potential $U(x)$, the level of spin velocity $\omega$, and level of energy from the external sources.

On the contrary, people's actions are based on the experience of different societies over the entire history of humanity, as if most of the society know exactly what lies ahead and exactly what is needed to avoid potential problems. That is well beyond the classical model.

Such cases must be analyzed from the quantum perspective and are subject to future politiphysical research.

\section{ACKNOWLEDGMENTS}

The author is grateful to Igor Mandel, Peter Miller, Ronald Cherry, Gregory Viola, and Valentina Gindler for fruitful and stimulating discussions.

[1] G. Gindler, The Man-State Paradigm, American Thinker, January 20, 2019

[2] I. Mandel, D. Kuznetsov, Statistical and physical paradigms in the social sciences. Model Assisted Statistics and Applications 4, 39-62 (2009)

[3] V.M. Yakovenko, Monetary economics from econophysics perspective, Eur. Phys. J. Special Topics 225, 3313-3335 (2016)

[4] L.D. Landau, E.M. Lifshitz, Course of Theoretical Physics, Vol. 1 (ButterworthHeinemann, $3^{\text {rd }}$ edition, 1976)

[5] I.S. Gradshteyn, I.M. Ryzhik, Tables of Integrals, series, and Products, (Academic Press, $5^{\text {th }}$ edition, 1994)

[6] S. Foss, S. Zachary, D. Korshunov, An Introduction to Heavy-Tailed and Subexponential Distributions, (Springer, 2011)

[7] https://en.wikipedia.org/wiki/Laffer_curve

[8] G. Aly, Hitler's Beneficiaries, (Picador Paper, 2008)

[9] B.K. Chakrabarti, A. Chakraborti, A. Chatterie (eds.), Econophysics and sociophysics: trends and perspectives (Wiley-VCH, 2006) 
INTRODUCTION TO POLITIPHYSICS

[10] F. Lillo, Econophysics and the Challenge of Efficiency, (Wiley Periodicals, Inc., Vol. 14, No. 3, 2008 - Econophysics)

[11] A. Drăgulescu, V.M. Yakovenko, Evidence for the exponential distribution of income in the USA, Eur. Phys. J. B 20, 585-589 (2001)

[12] B. K. Chakrabarti, A. Chakraborti, S. R. Chakravarty, A. Chatterjee, Econophysics of Income and Wealth Distributions (Cambridge University Press, 2013) 